\title{
Simulation of Flame Acceleration and DDT
}

\author{
Knut Vaagsaether \\ Department of Process, Energy and Environmental Technology, University College of Southeast Norway, Porsgrunn, \\ Norway, knutv@usn.no
}

\begin{abstract}
This paper presents a combustion model and a simulation method for modeling flame acceleration (FA) and deflagration to detonation transition (DDT) in a premixed gas. The method is intended to produce the most important effects in FA and DDT without resolving the flame front on the computational mesh. The simulations presented here are of stoichiometric hydrogen-air mixtures in a channel with repeated obstacles. The channel is $2 \mathrm{~m}$ long and $110 \mathrm{~mm}$ wide, with a height of either $20 \mathrm{~mm}$ or $40 \mathrm{~mm}$. The obstacles gives a blockage ratio of 0.5 . These values are the same as for experiments by other researchers and is used for comparison. The combustion model combines a turbulent burning velocity model and a two-step Arrhenius kinetic rate. The simulations show similar flame speeds and pressures as seen in experiments, and the process of DDT is shown to be caused by shock focusing and shock flame interactions. The simulations show that the quasi detonation regime is a series of transition to detonation events followed by failure of the detonation. Results from both 2D and 3D simulations are presented, since the 2D simulations show how the method can reproduce important effects.

Keywords: CFD, flame acceleration, DDT, detonation, hydrogen
\end{abstract}

\section{Introduction}

Simulations of strong flame acceleration and deflagration to detonation transition (DDT) in gaseous mixtures are important for understanding the propagation of gas explosions. Simulation tools are also important for risk assessment in industries where gas explosions might occur. Gamezo et al. (2007) presented simulations of flame acceleration and DDT in obstructed channels (Gamezo et al., 2007), in which a single step Arrhenius reaction rate describes the chemistry. The computational mesh resolution in their simulations was approximately 100th of a flame thickness. At a larger scale, such resolutions might be impossible to accomplish. Strong flame acceleration in a complex geometry is usually a product of classical fluid mechanical instabilities such as turbulence, Kelvin-Helmholtz, Rayleigh-Taylor, and Richtmyer-
Meshkov. Other important effects are the flamegeometry and pressure wave-geometry interactions, in which the flame surface area increases or the reactants are compressed. With sufficient compression, the hot pockets in the reactants might ignite and possibly lead to DDT. Lee and Moen (1980) and Lee et al. (1985) described different propagation regimes in obstructed channels, including: i) choked flow, ii) quasidetonation, and iii) Chapman-Jouguet (CJ) detonation. The choked flow regime is a deflagration in which the expansion of gas over an obstacle produces high flame speeds. In the quasi-detonation regime, the flame undergoes a transition to detonation followed by a failure of detonation due to diffraction. This DDT and failure process is repeated to produce higher average flame speeds than in the choking regime but lower than the CJ detonation speed. Thomas (2012), Shepherd (2009), Ciccarelli and Dorofeev (2008), and Shepherd and Lee (1992) have written excellent reviews on flame acceleration and DDT. The objective of this work is to develop a simulation method to predict the strong flame acceleration and DDT that can occur in gas explosions in channels with repeated obstacles. The focus of this paper is the development of a combustion model that can reproduce the phenomena seen in flame acceleration and DDT on an under-resolved computational mesh. More details on the method presented in this paper are described in Vaagsaether (2010).

\section{Combustion Model}

The combustion model combines a turbulent burning velocity model with a chemical kinetic rate model. A two-step reaction model is used for the chemical kinetic rate in all cases. Two reaction variables describe the total reaction, one for the initiation and chain branching reactions, and one for the termination rates. It is assumed that the initiation and branching reactions are isothermal. Three species are conserved as two reaction progress variables. These species are reactants, intermediates (radicals), and products. Here, the two progress variables are called $\alpha$ and $\beta$ and the conservation equations are provided in (1) and (2). 


$$
\begin{aligned}
& \frac{\partial \rho \alpha}{\partial t}+\nabla \cdot(\rho \vec{U} \alpha)=\dot{\Omega} \\
& \frac{\partial \rho \beta}{\partial t}+\nabla \cdot(\rho \vec{U} \beta)=\dot{\omega}
\end{aligned}
$$

Since the reaction described by (1) is assumed isothermal and the products of that reaction are assumed to be radicals, the reaction term for (1) is given by the induction time as seen in (3).

$$
\dot{\Omega}=\frac{\rho}{\tau_{\text {ind }}}
$$

Equation (4) shows a typical form for the induction time model, where $[\mathrm{F}]_{0}$ and $\left[\mathrm{O}_{2}\right]_{0}$ are the non-reacted fuel and oxygen concentrations, $A_{\text {ind }}$ is a preexponential factor, and $\mathrm{T}_{\mathrm{a} \text {,ind }}$ is an activation temperature.

$$
\begin{aligned}
& \tau_{\text {ind }}=A_{\text {ind }}[F]_{0}^{x}\left[O_{2}\right]_{0}^{y} \exp \left(T_{\text {a,ind }} / T\right) \\
& \dot{\omega}=\max \left[\left(\rho_{u} S_{T}|\nabla \beta|\right),\left(\dot{\omega}_{k}\right)\right]
\end{aligned}
$$

The reaction term in (2) accounts for both turbulent reactions and chemical kinetics. The rate is the maximum reaction rate of a turbulent burning velocity model and an Arrhenius-type kinetic rate. This term is seen in (5) where the first part of the rate is a typical flame density model and the second part is a Arrhenius kinetic rate.

$$
\dot{\omega}_{k}=\left\{\begin{array}{cl}
A p^{n} T^{m} \beta^{\circ} \exp \left(-\frac{T_{a}}{T}\right) & \text { if } \alpha \geq 1 \\
0 & \text { if } \alpha<1
\end{array}\right.
$$

Since the turbulent burning velocity model contains all reactions in the flame front, unlike the kinetic term it is not dependent on $\alpha$. A typical form of the kinetic rate of $\beta$ is shown in (6), where $A$ is a pre-exponential factor and $T_{a}$ is an activation temperature. In this model the $\alpha$-reaction must finish before the Arrhenius rate in the $\beta$-reaction can start, as seen in (6). In the unreacted unheated gas, $\alpha$ is 0 and increases with increasing temperature. In gas mixtures with a low temperature in the unreacted gas, the turbulent rate is dominant and the model behaves like a turbulent burning rate model. When the temperature in the reactants increases and the induction time becomes sufficiently small, the kinetic rate in the $\beta$ equation will start to influence the total rate. This property of the model can capture the effect of ignition by shock compression of the reactants.

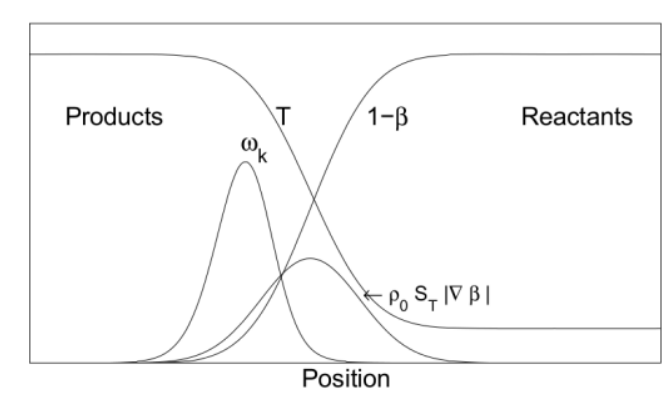

Figure 1: Schematic representation of the reaction rates with $\beta$ and temperature curves. The Turbulent rate highest at the highest gradient of the reaction rate variable. The Arrhenius rate is highest towards the product side.

Figure 1 shows a schematic representation of the combustion model with the two reaction rates for turbulent combustion and the Arrhenius rate. When the temperature in the reactants increases, the peak in the Arrhenius rate moves toward the reactant side.

\section{Geometry Simulation set-up}

Figure 2 shows a schematic of the experimental setup from Teodorczyk (2007), which is also the present simulation domain. The channel is $2 \mathrm{~m}$ long, $110 \mathrm{~mm}$ wide, and closed in all directions. The blockage ratio for all experiments is 0.5 . The ignition of the stoichiometric hydrogen-air mixture at atmospheric pressure and $293 \mathrm{~K}$ occurs at the center of one end wall. Results from the simulations with channel heights of $20 \mathrm{~mm}$ and $40 \mathrm{~mm}$ are presented here. These simulations are for a 2D geometry and show that the model handles the most important effects of flame acceleration and transition to detonation in this type of geometry. Two different mesh sizes, $1 \mathrm{~mm}$ and 0.5 $\mathrm{mm}$, are tested, but most of the results presented here use the $1 \mathrm{~mm}$ mesh. Some results from the 3D simulations are shown and compared with the experimental results. The 3D simulations use a constant $1 \mathrm{~mm}$ mesh. Since the real geometry is $3 \mathrm{D}$, the shock focusing and reflections behave differently than they would in 2D.

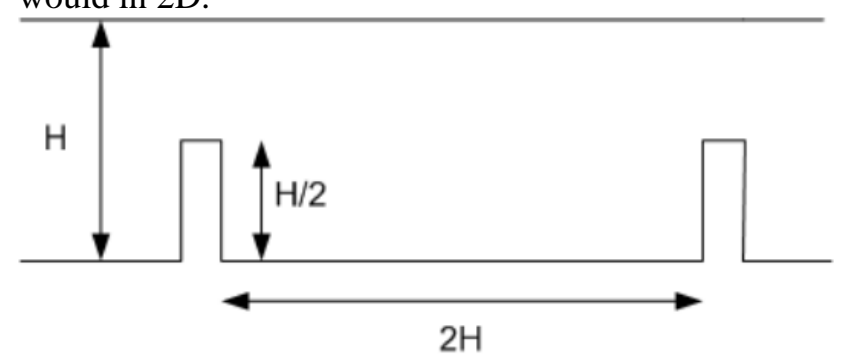

Figure 2: Experimental set-up of Teodorczyk with channel height and distance between obstacles. 


\subsection{1 Simulation set-up}

A second-order centered TVD method (FLIC) solves the transport equations for mass, momentum and energy and the gas is modeled as an ideal gas. This method is described in Toro (1999). A turbulence model is used since the flow is turbulent and the turbulent length scales are smaller than the mesh size. Equation (7) shows the one-equation model for the turbulent kinetic energy used in the simulations, where [R] is the Reynolds stress, [S] is the mean strain rates, $\mathrm{C}_{\mathrm{e}}$ is a constant set to 0.92 , and the turbulent length scale 1 is set to be the mesh size. The notation ":" is the Frobenius inner product of two matrices.

$$
\rho \frac{D k}{D t}=\nabla \cdot\left(\mu_{t} \nabla k\right)-[R]:[S]-C_{e} \rho \frac{k^{1.5}}{l}
$$

The turbulent burning velocity is calculated from a model presented in (Flohr and Pitsch, 2000) and is shown in (8).

$$
S_{T}=S_{L}\left(1+A \frac{(R e \cdot P r)^{0.5}}{D a^{0.25}}\right)
$$

A is a model constant and is set to $0.52, \mathrm{Re}$ is the turbulent eddy Reynolds number, $\operatorname{Pr}$ is the Prandtlnumber, Da is the turbulent eddy Dahmkohler number and the velocity fluctuation is calculated as $u^{\prime}=(2 / 3$ k) $)^{0.5}$.

The induction time model for hydrogen-air is presented in Sichel et al. (2002), where $\mathrm{A}_{\text {ind }}=1.1085 \cdot 10^{10} \mathrm{~Pa} \cdot \mathrm{s} / \mathrm{K}$ for a stoichiometric mixture.

$$
\begin{gathered}
\tau_{\text {ind }}=A_{\text {ind }} \frac{T}{p} \exp (B) \\
B=-35.17+\frac{8530.6}{T}+7.22 \cdot 10^{-11}\left(\frac{p}{p_{0}}\right)^{2} \exp \left(\frac{21205}{T}\right)
\end{gathered}
$$

The rate of the exothermic reaction was presented in (Korobeinikov, 1972) where $A_{\beta}=1.04 \cdot 10^{-5}$ and $\mathrm{E}_{\mathrm{a}}=2000 \mathrm{~K}$.

$$
\frac{\partial \beta}{\partial t}=A_{\beta} p^{2} \exp \left(-\frac{E_{a}}{T}\right)\left(\beta^{2}-(1-\beta)^{2} \exp \left(-\frac{Q}{R T}\right)\right)
$$

The laminar burning velocity is taken from a model presented in Iijima and Takeno (1984), where $\mathrm{p}_{0 \mathrm{~s}}=101325 \mathrm{~Pa}$ and $\mathrm{T}_{0 \mathrm{~s}}=291 \mathrm{~K}$.

$$
S_{L}=S_{L, 0}\left(1+X \cdot \log _{10}\left(\frac{p}{p_{0 s}}\right)\right)\left(\frac{T}{T_{0 s}}\right)^{Y}
$$

$$
\begin{gathered}
S_{L, 0}=2.98-(\varphi-1.7)^{2}+0.32(\varphi-1.7)^{3} \\
X=0.43+0.003(\varphi-1) \\
Y=1.54+0.026(\varphi-1)
\end{gathered}
$$

The total energy per volume is:

$$
E_{\text {tot }}=\frac{p}{\gamma-1}+\frac{1}{2} \rho \vec{U} \cdot \vec{U}+\rho(1-\beta) Q
$$

The properties $\mathrm{Q}=3.2 \mathrm{MJ} / \mathrm{kg}, \gamma_{\mathrm{u}}=1.402, \gamma_{\mathrm{b}}=1.242$ are used for the stoichiometric mixture of hydrogen-air, where the subscripts $u$ and $b$ indicate the unburned and burned states.

\section{Results and discussion, 2D simulation}

To demonstrate how the simulation method handles shock ignition and DDT. Two-dimensional simulations are presented and discussed in this section. These results are presented as contour plots of the density gradients as well as plots of the flame speeds along the channel length just below the top wall. Fig. 3 shows the flame speed along the length of the $40 \mathrm{~mm}$ high channel. From ignition, the flame speed increases as the flame passes the obstacles. The expansion of the gas across the obstacles produces high flame speed and increases the burning rates, which in turn produce a shock wave traveling in front of the flame. Figure 4 shows the process of DDT in the $40 \mathrm{~mm}$ channel. When the shock passes an obstacle, a diffracted shock front reflects at the bottom wall and creates a Mach stem. Both the leading shock and the Mach stem reflect at the obstacles and are focused in the corner between the bottom wall and the obstacle. They ignite the gas behind the focused shock to send a strong shock wave into the products. This shock wave diffracts over the obstacles and reflects at the top wall. The reflected and diffracted shock interacts with the flame from the product side and accelerates the flame, and it may even heat the reactants in the front of the flame to cause DDT. 


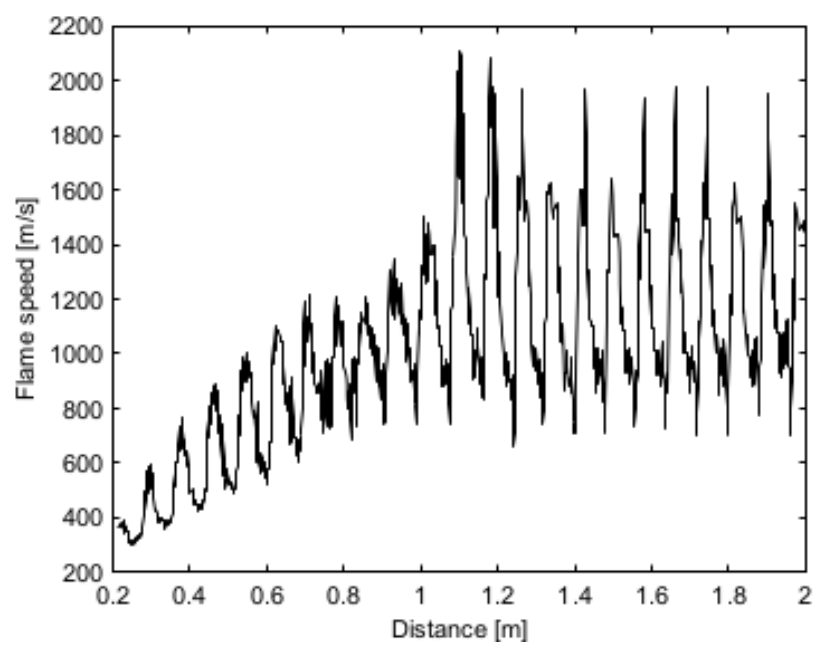

Figure 3: Simulated flame speed as a function of time for the $40 \mathrm{~mm}$ channel with repeated obstacles with $1 \mathrm{~mm}$ mesh. Stoichiometric hydrogen-air at $293 \mathrm{~K}$ and $1 \mathrm{~atm}$.

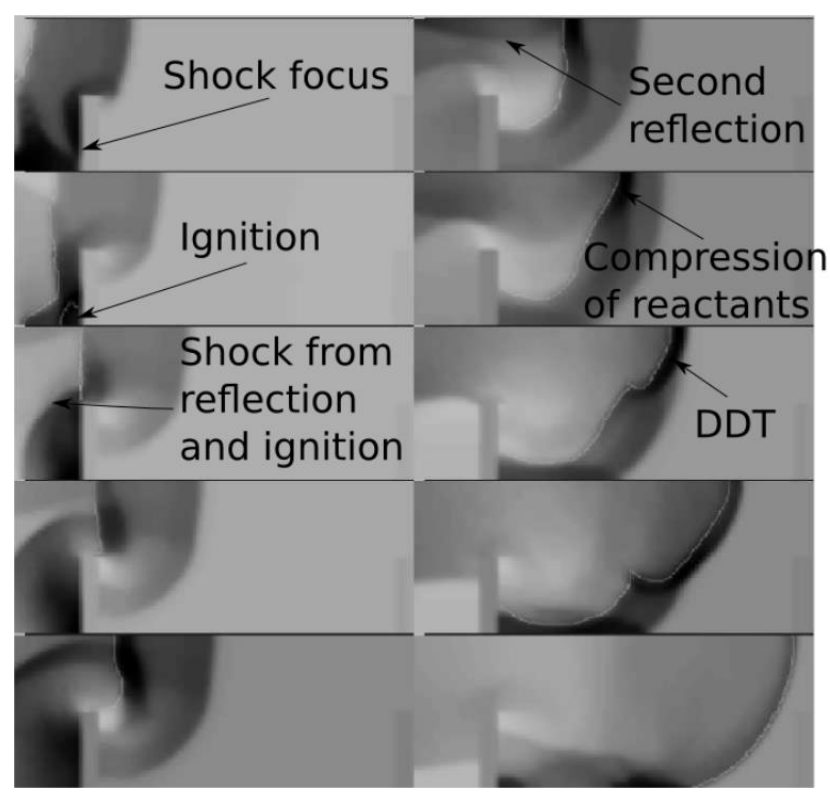

Figure 4: 2D simulation of $40 \mathrm{~mm}$ channel height showing density gradients of shock-flame-obstacle interactions where transition to detonation occurs.

When the detonation in the $40 \mathrm{~mm}$ channel propagates past an obstacle, the shock diffracts and the detonation fails, as can be seen in Figure 5. Due to the diffraction over an obstacle, the flame does not propagate as a CJ detonation. The average flame speed after this point is about $1400 \mathrm{~m} / \mathrm{s}$ and can be interpreted as the quasidetonation regime. In the experiments, there is significant scatter in the locations of the first DDT. The average flame speed in the experiments after this first transition is about $1250 \mathrm{~m} / \mathrm{s}$, but varies as much as 200 $\mathrm{m} / \mathrm{s}$. A similar process of DDT and failure is seen in high-speed photographs in experiments in Teodorczyk et al., (1988).
The simulations of the $20 \mathrm{~mm}$ channel show similar flame acceleration to what occurred in the $40 \mathrm{~mm}$ channel. Figure 6 shows the simulated flame speed along the channel length. After about $0.4 \mathrm{~m}$ distance from ignition, the flame reaches an average speed of over $1000 \mathrm{~m} / \mathrm{s}$, fluctuating between $1200 \mathrm{~m} / \mathrm{s}$ and 800 $\mathrm{m} / \mathrm{s}$, and it is described as the choking regime for this case. In the experimental results there is likely a transition to detonation around $0.7 \mathrm{~m}$, which is not seen in the simulation. But after about $1.0 \mathrm{~m}$ the flame speed is on average constant around $1000 \mathrm{~m} / \mathrm{s}$ in both the experiments and in the simulations. The coarse mesh is unable to resolve the smaller scales of the different instabilities important in flame acceleration, and these instabilities may form small hotspots that can cause DDT. The results of a grid sensitivity test are shown in Figure 7. The $40 \mathrm{~mm}$ channel is probably the most interesting case since it includes flame acceleration, DDT, and failure. The flame speed along the $40 \mathrm{~mm}$ channel is roughly the same for both mesh sizes.

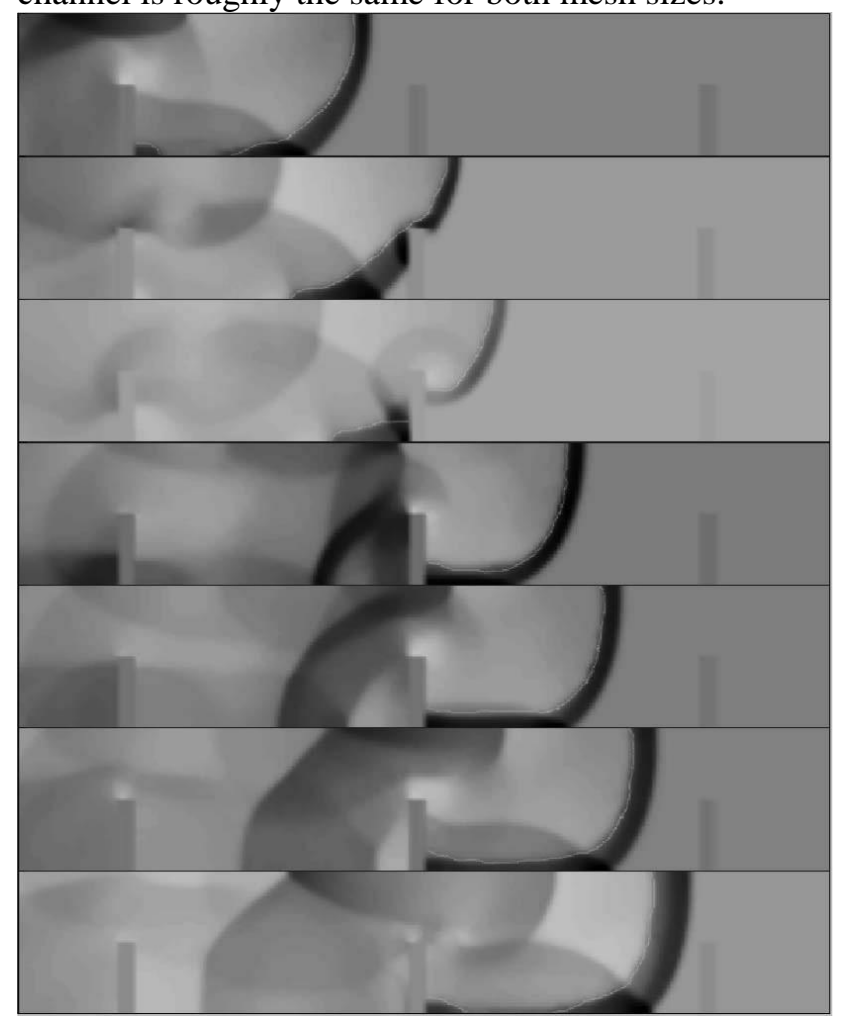

Figure 5: Simulated density gradients of shock-flameobstacle interactions for the $40 \mathrm{~mm}$ channel with repeated obstacles. The images show the failure of detonation. Stoichiometric hydrogen-air at $293 \mathrm{~K}$ and $1 \mathrm{~atm}$. 


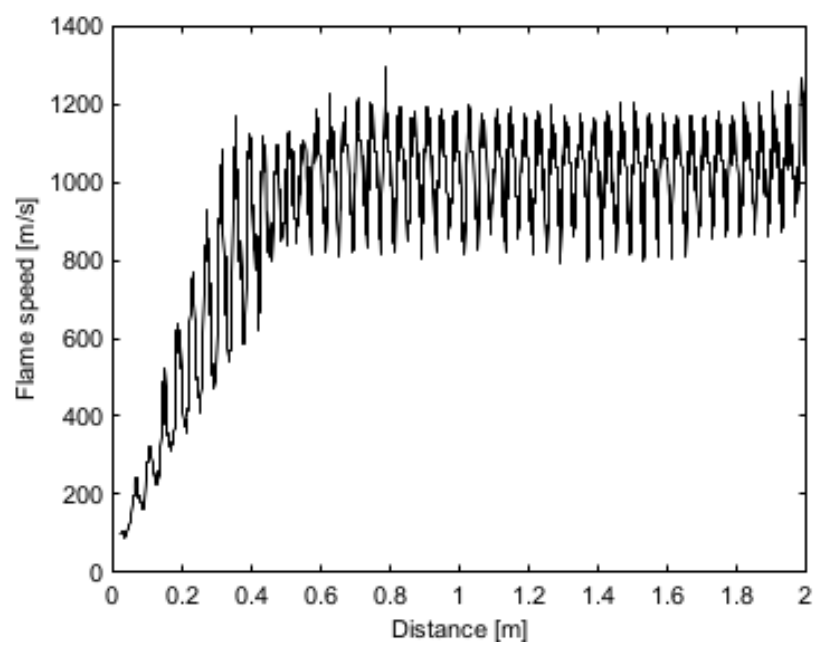

Figure 6: Simulated flame speed as a function of time for the $20 \mathrm{~mm}$ channel with repeated obstacles with $1 \mathrm{~mm}$ mesh. Stoichiometric hydrogen-air at $293 \mathrm{~K}$ and $1 \mathrm{~atm}$.

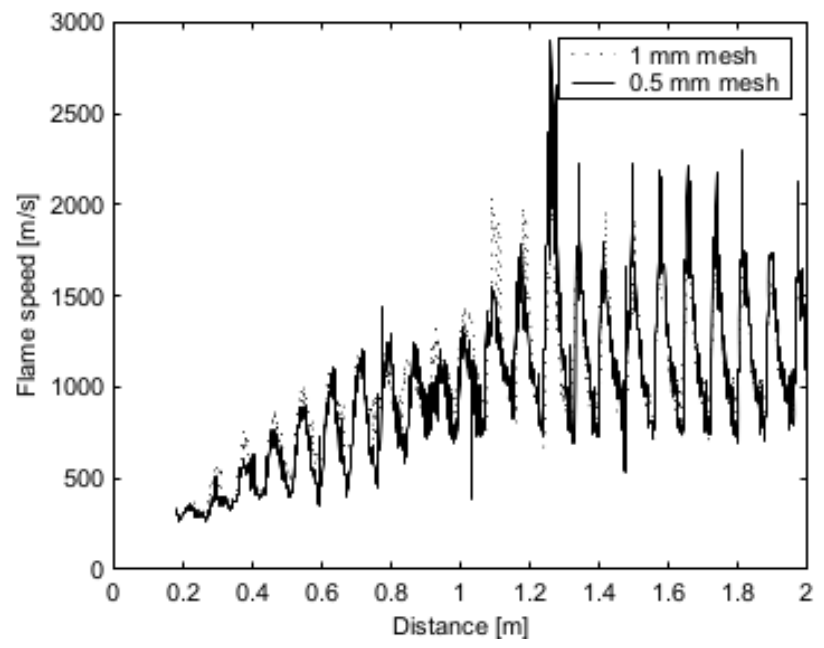

Figure 7: Grid sensitivity for the $40 \mathrm{~mm}$ channel with repeated obstacles for $1 \mathrm{~mm}$ mesh and $0.5 \mathrm{~mm}$ mesh. Stoichiometric hydrogen-air at $293 \mathrm{~K}$ and $1 \mathrm{~atm}$.

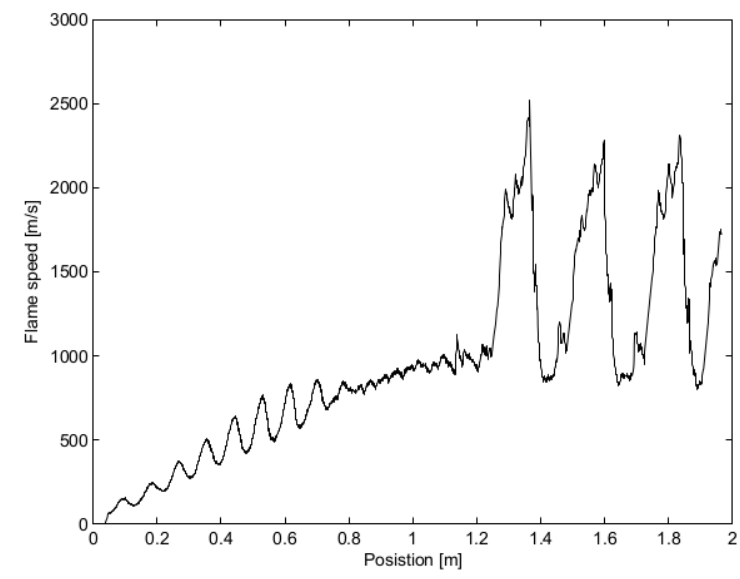

Figure 8: 3D simulation with $1 \mathrm{~mm}$ mesh of the flame speed along the center of the channel top wall for the 40 mm channel with repeated obstacles. Stoichiometric hydrogen-air at $293 \mathrm{~K}$ and $1 \mathrm{~atm}$.

\section{Results and discussion, 3D simulation}

Figure 8 shows the simulated flame speed along the channel. Figure 9, Figure 10 and Figure 11 show the experimental pressure histories at $795 \mathrm{~mm}, 875 \mathrm{~mm}$, and $955 \mathrm{~mm}$ from ignition, respectively, as well as the simulated pressure three obstacle distances farther down the channel. The experimental pressure records are extracted from the image files in Teodorczyk (2007) by a simple code. The accuracy of the extraction is not validated but it should reproduce the same curves as in the paper. The simulated time is set to match the strong pressure peak in Figure 9, since this peak is thought to be due to the initiation of the detonation.

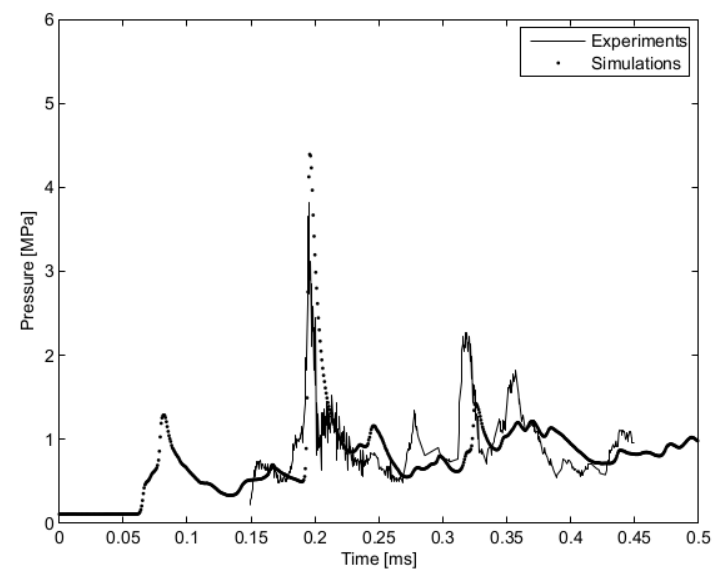

Figure 9: Experimental and simulated pressure history in the $40 \mathrm{~mm}$ channel with repeated obstacles.

Stoichiometric hydrogen-air at $293 \mathrm{~K}$ and $1 \mathrm{~atm}$. The pressure transducer is $795 \mathrm{~mm}$ from ignition; the transducer in the simulation is placed three obstacle distances farther from ignition. 


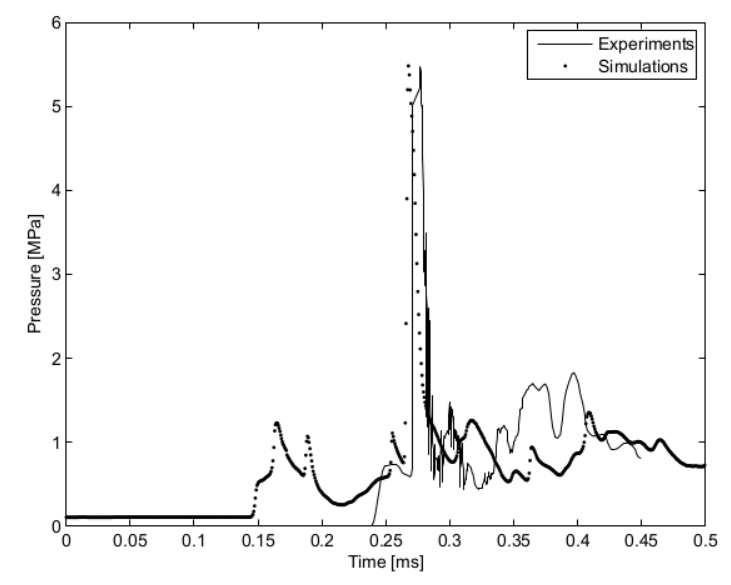

Figure 10: Experimental and simulated pressure history in the $40 \mathrm{~mm}$ channel with repeated obstacles. Stoichiometric hydrogen-air at $293 \mathrm{~K}$ and $1 \mathrm{~atm}$. The pressure transducer is $875 \mathrm{~mm}$ from ignition; the transducer in the simulation is placed three obstacle distances farther from ignition.

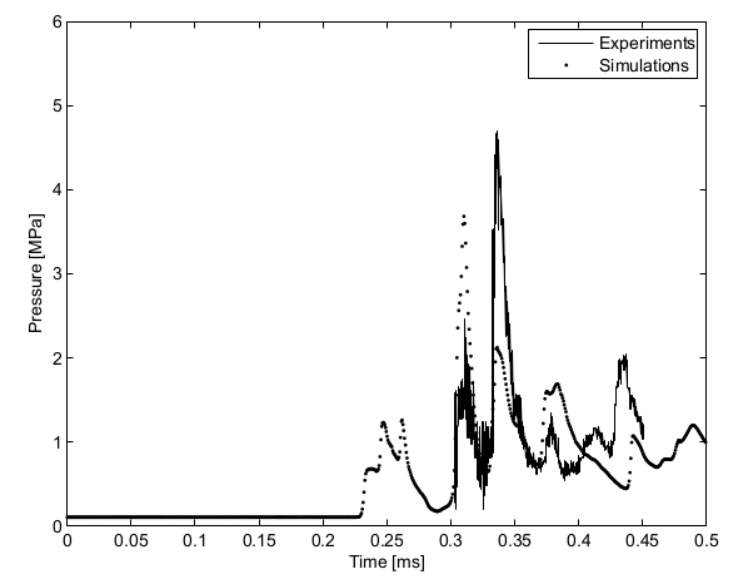

Figure 11: Experimental and simulated pressure history in the $40 \mathrm{~mm}$ channel with repeated obstacles. Stoichiometric hydrogen-air at $293 \mathrm{~K}$ and $1 \mathrm{~atm}$. The pressure transducer is $955 \mathrm{~mm}$ from ignition; the transducer in the simulation is placed three obstacle distances farther from ignition.

The pressure histories from the simulation are taken at a transducer position that is three obstacle distances farther from ignition. Since there is significant scatter in the experiments, the position of the first DDT is difficult to match with the experimental pressure data. The distance between the first pressure rise and the shock from the initiation of the detonation is longer in the simulations than in the experiments. Since the simulated pressure is shifted three obstacle distances, the leading shock has propagated farther from the flame. The coarse resolution of the mesh does not capture all the hotspots. This might average out small areas of high temperature to a lower temperature, whereas the hotspots may ignite and lead to detonation in the experiments. Compared with the 2D simulation, the 3D simulation predicts the first DDT one obstacle later. This might be because any strong shock produced from focusing may propagate in three directions, compared with two directions in the $2 \mathrm{D}$ case; furthermore, a simulated hotspot in $2 \mathrm{D}$ may lead to transition while in 3D it weakens faster and may not cause transition. Another reason might be that in 2D, the gas is ignited in the entire width of the channel and the flame propagates cylindrically and not spherically, so that the position is moved to where a sufficiently strong shock wave is formed. The $3 \mathrm{D}$ simulation does not show the same frequency of the pressure oscillations as with the 2D simulation. The flame propagates with the detonation velocity for the length of three obstacles, compared with only one for the 2D simulation. The shock diffraction is not as critical for the $3 \mathrm{D}$ simulation since the propagating detonation front is not planar. As the detonation passes the obstacle, parts of the detonation fail and cause transverse waves that keep the detonation going as a CJ detonation. Previous work on simulation of transition to detonation has been performed with much finer mesh (Gamezo et. al., 2007) but with simpler chemistry. The transition phenomena can be predicted with the present method even when the mesh size is larger than the flame thickness.

\section{Conclusions}

Simulations of flame acceleration and DDT using the present method reproduce the main effects seen in experiments of flame acceleration in channels with repeated obstacles. Shock focusing and reflections are the most important sources for producing hotspots that lead to the onset of detonation in channels with repeated obstacles. The same processes that led to detonation in the experimental results in Teodorczyk (2007) and Teodorczyk et al. (1988) are seen in the present results. The $3 \mathrm{D}$ simulation shows similar behavior to the 2D simulation where DDT occurs, and the flame propagates in the quasi-detonation regime. The simulated initiation and failure of detonation shows that this geometry with a point ignition behaves three-dimensionally, and the details are handled differently for 2D and 3D. The coarse mesh might be the reason for the difference between the simulations and experiments. Since the coarse mesh averages the flame over a few millimeters, the details in the formation of hotspots and the diffraction of the front are not captured. The experiments show significant scatter in the position of the DDT, and it is difficult to say how good the predictions are on that account.

\section{References}

G. Ciccarelli, and S. Dorofeev. Flame Acceleration and Transition to Detonation in Ducts. Progress in Combustion Science, 34(4): 499-550, 2008. 
P. Flohr, and H. Pitsch. A turbulent flame speed closure model for LES of industrial burner flows. Centre for Turbulent Research Proceedings of the Summer Program, 2000.

V. N. Gamezo, T. Ogawa, and E. S. Oran. Numerical simulations of flame propagation and DDT in obstructed channels filled with hydrogen-air mixures. In Proceedings of the Combustion Institute 31: 2463-2471, 2007.

T. Iijima, and T. Takeno. Effects of temperature and pressure on burning velocity. Proceedings of the Faculty of Engineering, Tokai Univ. Vol. X:53-67, 1984.

V. P. Korobeinikov, V. A. Levin, V. V. Markov, and G. G. Chernyi. Propagation of blast in a combustible gas. Astronautica Acta, 17: 529-537, 1972.

J. H. S. Lee, and I. O. Moen. The mechanism of transition from deflagration to detonation in vapor cloud explosions. Progress in Energy Combustion Science 6: 359-389, 1980.

J. H. Lee, R. Knystautas, and C. K. Chan. Turbulent flame propagation in obstacle filled tubes. In Proceedings of the Combustion Institute. 20: 1663-1672, 1985.

J. E. Shepherd, and J. H. S. Lee. On the transition from deflagration to detonation. In: M. Y. Hussaini, A. Kumar and R. G. Voigt, Editors, Major research topics in Combustion, 1992 .
J. E. Shepherd. Detonation in gases. In Proceedings of the Combustion Institute 32: 83-98, 2009.

M. Sichel, N. A. Tonello, E. S. Oran, and D. A. Jones. A two-step kinetics model for numerical simulation of explosions and detonations in $\mathrm{H} 2-\mathrm{O} 2$ mixtures. Proceedings of the Royal Society A. 458: 49-82, 2002.

A. Teodorczyk, J. H. S. Lee, and R. Knystautas. Propagation Mechanism of Quasi-Detonations. In Proceedings of the Combustion Institute 22: 1723-1731, 1988.

A. Teodorczyk. Scale effects on hydrogen-air fast deflagrations and detonations in small obstructed channels. Journal of Loss Prevention in the Process Industries, 21(2): 147-153, 2007. doi:10.1016/j.jlp.2007.06.017.

G. O. Thomas. Some observations on the initiation and onset of detonation. Philosofical Transactions of the Royal Society A. 370(1960): 715-739, 2012.

E. F. Toro. Riemann solvers and Numerical methods for fluid dynamics. Springer-Verlag Berlin Heidelberg, 1999, (ISBN 3-540-65966-8).

K. Vaagsaether. Modelling of gas explosions. PhD Thesis, 2010, Telemark Open Research Archive (http://teora.hit.no/dspace/), http://hdl.handle.net/2282/1113. 